\title{
Ammonium Acetate Supplement Strategy for Enhancement of Chaetominine Production in Liquid Culture of Marine-Derived Aspergillus fumigatus CY018 ${ }^{\mathrm{s}}$
}

\author{
Chang-Qing Liu, Xing-Chen Wei, Fa-Liang An, and Yan-Hua Lu*
}

State Key Laboratory of Bioreactor Engineering, East China University of Science and Technology, Shanghai, P.R. China

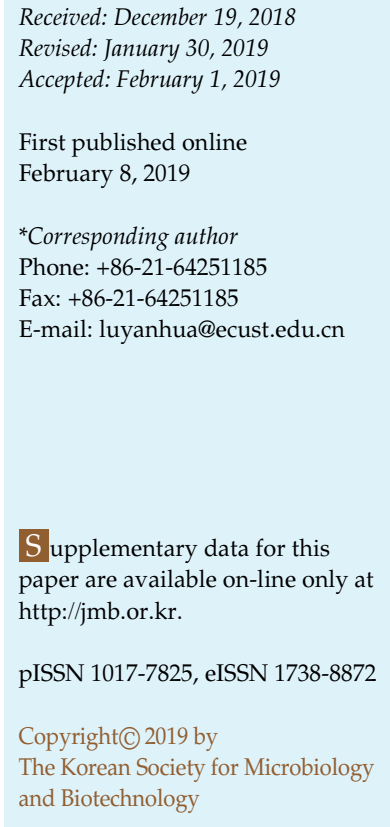

Pharmacological research on (CHA), a marine-derived quinazolinone alkaloid with significant cytotoxic activity, is restricted by low yields and is a problem that needs to be settled urgently. In this work, the selection of additional nitrogen sources and the optimization of additional concentrations and longer fermentation times using ammonium acetate, were investigated. CHA production was optimized to $62.1 \mathrm{mg} / 1$ with the addition of $50 \mathrm{mM}$ ammonium acetate at $120 \mathrm{~h}$ of the fermentation in the shaker flask. This feeding strategy significantly increased 3deoxy-arabino-heptulosonate-7-phosphate synthase activity and transcript levels of critical genes (laeA, dahp, and $\operatorname{trpC}$ ) in the shikimate pathway compared with the non-treatment group. In addition, the selection of the feeding rate $(0.01$ and $0.03 \mathrm{~g} / 1 \cdot \mathrm{h})$ was investigated in a 5-L bioreactor. As a result, CHA production was increased by $57.9 \mathrm{mg} / 1$ with a $0.01 \mathrm{~g} / \mathrm{l} \cdot \mathrm{h}$ ammonium acetate feeding rate. This work shows that the strategy of ammonium acetate supplementation had an effective role in improving CHA production by Aspergillus fumigatus CY018. It also shows that this strategy could serve as an important example of large-scale fermentation of a marine fungus in submerged culture.

Keywords: Chaetominine, marine-derived fungus, ammonium acetate, feeding strategy, Aspergillus fumigatus

\section{Introduction}

Marine resources have received the attention of many scholars owing to the abundance of bioactive compounds with unique chemical structures produced by marine organisms [1, 2]. Marine-derived microorganisms, a renewable, prolific and efficient resource, produce various secondary metabolites with remarkable bioactivities, such as antitumor, antibacterial, and anti-inflammatory effects [3, 4]. However, the research and development of secondary metabolites within a distinct framework in the pharmacodynamics field are seriously limited by low yields $[5,6]$. Therefore, effective biotechnological strategies imminently need to be exploited to enhance the production of target compounds by the fermentation of marine microbes.

Many secondary metabolites from marine symbiotic microbes possess excellent bioactivity and some have been developed into drugs for the treatment of various diseases [7, 8]. Aspergillus fumigatus CY018, a symbiotic fungus of marine crab, produces many valuable metabolites with antifungal, anticancer and cytotoxic activities $[9,10]$. Chaetominine (CHA), isolated and identified from A. fumigatus CY018, is a quinazolinone alkaloid (Fig. 1) with significant anticancer activity against the human leukemia K562 and colon cancer SW1116 cell lines. Yao and co-workers found a mechanism whereby $\mathrm{CHA}$ treatment induced apoptosis in human leukemia K562 cells via the mitochondrial pathway [11, 12]. However, an effective regulation strategy for improving the production of $\mathrm{CHA}$ is not available thus far, which limits further research about the pharmacology and pharmacodynamic mechanism of the anticancer activity of $\mathrm{CHA}$.

The supplementation of medium components as a significantly effective strategy has been widely applied in 


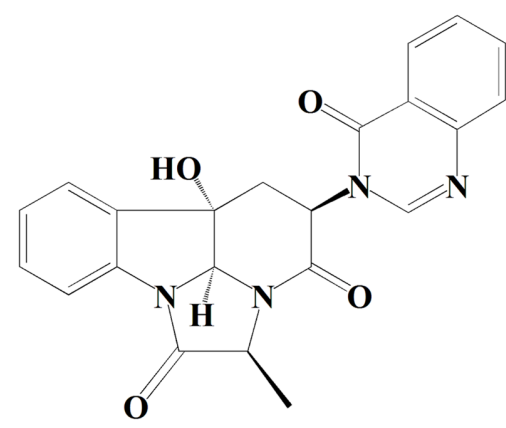

Fig. 1. Chemical structure of chaetominine.

the fermentation process for enhancing production of target metabolites. A nitrogen source as an important nutritional component affects the growth of microorganisms and the synthesis of secondary metabolites [13]. On one hand, the effect of limiting nitrogen can improve the production of secondary metabolites. Wang et al. found that limiting ammonium sulfate in culture medium could improve the production of pullulan by Aureobasidium pullulans [14]. On the other hand, the supplementation of nitrogen can also effectively promote the biosynthesis of target compounds. For instance, a feeding strategy with a nitrogen source was beneficial for cell growth and curdlan production during the early fermentation stage of Rhizobium radiobacter [15].

CHA was speculated to be biosynthesized via the shikimate pathway in a previous work. Although the detailed polymerization of alanine, anthranilic acid and tryptophan is unclear, the early steps of the CHA biosynthetic pathway were verified as involving the shikimate pathway in our previous work [16]. There are some key sites for control in the shikimate pathway, such as 3-deoxy-arabino-heptulosonate7-phosphate synthase (DAHP, dahp), chorismate synthase $(c s)$ and anthranilate synthase $(\operatorname{trp} C)$ [17-19]. In addition, lae $A$, a global regulator, plays an important regulation role in the biosynthesis of secondary metabolites, such as the effect of deletion/overexpression of laeA in Aspergillus app. [20]. Thus, the transcriptional expression of these key genes, lae $A$, dahp, cs and $\operatorname{trp} C$, might have a promoting role in the biosynthesis of CHA by way of a nitrogen source feeding strategy. In addition, the transcript levels of critical genes are verified to enhance the production of target compounds [21,22]. For example, limiting glutamine (a nitrogen source) improves the transcriptional expression of important related genes and the yield of ganoderic acids in Ganoderma lucidum [23].

Feeding nutrients into the culture broth is a typical strategy for enhancement of the target compounds during fermentation, especially a nitrogen source. Moreover,
Zhang et al. found that the optimization of nitrogen sources significantly influences CHA biosynthesis. Although the concentration of the nitrogen source was optimized in the previous work [24], the CHA production was still low and this problem urgently requires solving.

In the present work, the supplementation effect of different nitrogen sources in the broth was first investigated. Then, the optimal concentration and time of feeding ammonium acetate were determined in the shake flask. Metabolic parameters (dry cell weight (DCW), residual sugar, CHA production and the activity of DAHP synthase) and transcript levels of critical genes (laeA, dahp, cs, and $\operatorname{trpC}$ ) were detected with the optimal fermentation conditions. Furthermore, different feeding rates of ammonium acetate were studied in a 5-L bioreactor. The results of this work are valuable for enhancing the production of target compounds by feeding nutrients into the submerged fermentation process.

\section{Materials and Methods}

\section{Microorganism}

The CHA-producing A. fumigatus CY018 utilized in this work was kindly provided by Nanjing University (China). Mycelia were first cultured on potato dextrose agar (PDA) medium for seven days at $28^{\circ} \mathrm{C}$ in the constant temperature incubator then subcultured monthly [25].

\section{Fermentation Conditions}

A $1.0 \mathrm{~cm}^{2}$ agar tablet was cut from cultivated PDA and transferred to a $500 \mathrm{ml}$ shake flask containing $200 \mathrm{ml}$ potato dextrose broth (PDB). After inoculation, the flask was cultured as seeds at $180 \mathrm{rpm}$ and $28^{\circ} \mathrm{C}$ for $72 \mathrm{~h}$ in an oscillating constant temperature shaker. Then, a $250 \mathrm{ml}$ shake flask with $50 \mathrm{ml}$ fermentation medium was inoculated with $7 \mathrm{ml}$ of seeds for culturing at $180 \mathrm{rpm}$ and $28^{\circ} \mathrm{C}$ for $336 \mathrm{~h}$ in an oscillating constant temperature shaker. The fermentation medium was composed of (g/l): sucrose, 100; ammonium acetate, 5; sodium tartrate, 2; sodium glutamate, $2.4 ; \mathrm{KH}_{2} \mathrm{PO}_{4}, 1.2 ; \mathrm{MgSO}_{4} \cdot 7 \mathrm{H}_{2} \mathrm{O}, 0.84$; and $\mathrm{FeSO}_{4} \cdot 7 \mathrm{H}_{2} \mathrm{O}, 0.016$.

\section{Optimization of the Nitrogen Source}

The effects of different nitrogen sources (ammonium sulfate, ammonium chloride, and ammonium acetate) with 5 and $10 \mathrm{mM}$ concentrations at $120 \mathrm{~h}$ of fermentation on CHA production and DCW were investigated. The effects on CHA production and DCW with ammonium acetate were further examined with different concentrations $(5,10,20,50$, and $100 \mathrm{mM})$ at $120 \mathrm{~h}$ during the fermentation. Then, ammonium acetate (screened concentration) was supplied at $72,120,168,216,264$, and $312 \mathrm{~h}$ in the fermentation process, respectively. The non-treatment groups were performed in cultivation medium without the addition of ammonium acetate. 


\section{Sampling and Analysis}

At the end of the fermentation process, DCW was determined to analyze the biomass of $A$. fumigatus. The anthrone-sulfuric acid method was utilized to measure the residual sugar of fermentation broth at different detection times. High-performance liquid chromatography was used to assay the content of CHA in the broth as described by Liu et al. [26]. One milliliter of broth was centrifuged and the underlying mycelium was analyzed for the activity of the key enzyme DAHP synthase in the shikimate pathway. The method of analyzing DAHP synthase was quoted in the published articles [16]. After the reaction was ended, the final solution was determined by measuring the decrease of PEP with a spectrophotometer at $549 \mathrm{~nm}$.

Measurement of the Expression of Key Enzyme Genes by Quantitative Reverse Transcription Polymerase Chain Reaction (qRT-PCR)

The TRIzol solution was employed to extract total RNA from the samples taken for all experiments. The RNA concentrations in the samples were determined using a spectrophotometer at $\mathrm{A}_{260 /}$ 280 . The method of Li et al. was utilized to detect mRNA expression levels of laeA, dahp, cs, trpC and actin [27]. Total RNA served as the initial template and the PremixScript Reagent Kit (Takara Bio, Japan) was used for reverse transcription. Premix Ex Taq II (Takara Bio) was utilized to determine mRNA levels of key genes according to the manufacturer's procedure. Primer pair sequences for qRT-PCR amplification of laeA, dahp, cs, $\operatorname{trp} C$, and actin are displayed in Table 1. The qRT-PCR was performed as displayed by the published paper [16].

\section{Batch-Fed Fermentation in a 5-L Bioreactor}

The research on feeding flow rate with ammonium acetate was worthy in the bioreactor. The feeding flow rates for ammonium acetate $(0.01 \mathrm{~g} / \mathrm{l} \cdot \mathrm{h}, 0.03 \mathrm{~g} / \mathrm{l} \cdot \mathrm{h}$ and non-treatment) on batch-fed fermentation were investigated in a 5 -L stirred bioreactor. A $1 \mathrm{~L}$ shake flask containing $400 \mathrm{ml}$ PDB was cultured on a shaker at $180 \mathrm{rpm}$ and $28^{\circ} \mathrm{C}$ for $72 \mathrm{~h}$ in an oscillating constant temperature shaker. Then, this was inoculated into the bioreactor containing

Table 1. Sequences of primer pairs for quantitative real-time RT-PCR (qRT-PCR) assay.

\begin{tabular}{|c|c|c|}
\hline Target gene & Primer name & Primer sequence $\left(5^{\prime}-3^{\prime}\right)$ \\
\hline \multirow[t]{2}{*}{ laeA } & laeA-forword & TTCTTTCGAGCTGCCGTCAA \\
\hline & laeA-reverse & TCCATGGTATGTTCGTCCGT \\
\hline \multirow[t]{2}{*}{ dahp } & dahp-forword & GACAAGGGCCCATCTTCACA \\
\hline & dahp-reverse & TGGCAGACGTGTTATAGCGG \\
\hline \multirow[t]{2}{*}{ CS } & cs-forword & CCCCCGAACAAATGAATCGC \\
\hline & cs-reverse & GGTACTTCTCCGCAATGGCT \\
\hline \multirow[t]{2}{*}{$\operatorname{trpC}$} & $\operatorname{trpC}$-forword & AGGTCGACGTTTTCGGTGAA \\
\hline & $\operatorname{trpC}$-reverse & AGTCACTTCAACCGCAGGAG \\
\hline \multirow[t]{2}{*}{ actin } & actin-forword & TTCGAGACCTTCAACGCTCC \\
\hline & actin-reverse & ATGGGGACAACGTGAGTGAC \\
\hline
\end{tabular}

2.6 L medium and fermented for $384 \mathrm{~h}$ at $28 \pm 0.2^{\circ} \mathrm{C}$ with a stirring speed of $300 \mathrm{rpm}$. Air flow was controlled at $1.5 \pm 0.1$ vessel volume/min during the fermentation process. Antifoam (a mixture of organic polyether dispersions) was added at $0.3 \%(\mathrm{v} / \mathrm{v})$ before autoclaving. Fermentation broth $(20 \mathrm{ml})$ was taken at intervals of $48 \mathrm{~h}$ for detecting the metabolic parameters.

\section{Statistical Analysis}

All experiments in the shake flasks were performed in triplicate. Error bars in the figures indicate the corresponding standard deviations. A value of $p<0.05$ was considered statistically significant. Origin 8 software (OriginLab, USA) was utilized to create the figures and CFX Manager (Bio-Rad, USA) was used to analyze the qRT-PCR data.

\section{Results and Discussion}

\section{Effects of Adding Different Nitrogen Sources}

Nutrients in the culture broth, especially nitrogen sources, played important roles in the biosynthesis of secondary metabolites. Moreover, Zhang et al. discovered that the effects of different nitrogen sources and concentrations on CHA production were obviously significant [24]. However, the production of CHA was also at a low level. Therefore, we fed the supernumerary nitrogen source for enhancement of CHA production. The effects of feeding 5 and $10 \mathrm{mM}$ of three different nitrogen sources (ammonium sulfate, ammonium chloride, and ammonium acetate) were investigated. As shown in Table 2, the production of CHA was $31.6 \pm 3.1$ and $25.4 \pm 1.6 \mathrm{mg} / 1$ with 5 and $10 \mathrm{mM}$ ammonium sulfate, respectively. This indicated that CHA production was decreased with an increasing amounts of ammonium sulfate, which implied that effect of ammonium sulfate addition was not significant on $\mathrm{CHA}$ production.

The production of CHA $(32.5 \pm 1.6$ and $34.5 \pm 3.2 \mathrm{mg} / \mathrm{l})$ was slightly increased with 5 and $10 \mathrm{mM}$ ammonium

Table 2. Effect of different nitrogen sources addition on CHA biosynthesis.

\begin{tabular}{lcc}
\hline \multicolumn{1}{c}{ Nitrogen source } & $\begin{array}{c}\text { Dry cell weight } \\
(\mathrm{g} / \mathrm{l})\end{array}$ & $\begin{array}{c}\text { Production of CHA } \\
(\mathrm{mg} / \mathrm{l})\end{array}$ \\
\hline Non-treatment group & $21.98 \pm 1.60$ & $29.27 \pm 3.04$ \\
$5 \mathrm{mM}$ ammonium sulfate & $19.43 \pm 2.04$ & $31.62 \pm 3.05$ \\
$10 \mathrm{mM}$ ammonium sulfate & $19.46 \pm 1.99$ & $25.35 \pm 1.55$ \\
$5 \mathrm{mM}$ ammonium chloride & $20.53 \pm 1.27$ & $32.52 \pm 2.11$ \\
$10 \mathrm{mM}$ ammonium chloride & $20.61 \pm 1.28$ & $34.54 \pm 3.17$ \\
$5 \mathrm{mM}$ ammonium acetate & $20.47 \pm 1.13$ & $32.73 \pm 1.25$ \\
$10 \mathrm{mM}$ ammonium acetate & $21.83 \pm 2.11$ & $42.52 \pm 2.26^{*}$
\end{tabular}

${ }^{*}$ Indicates statistical significance $(p<0.05)$ compared to the non-treatment group. 
chloride. The highest production of CHA was $42.5 \mathrm{mg} / 1$ with the addition of $10 \mathrm{mM}$ ammonium acetate in the selection experiments, in which production was $45.3 \%$ higher than the non-treatment group. Therefore, the addition of ammonium acetate played a significantly promoting role in CHA production, suggesting that feeding ammonium acetate might be an effective strategy for enhancement of CHA production.

\section{Concentration-and Time- Effects of Ammonium Acetate Addition on CHA Production}

Because feeding ammonium acetate was beneficial for increasing CHA production by $A$. fumigatus CY018, the optimal concentration and time of ammonium acetate supplementation were investigated. As shown in Fig. 2A, the effects of different concentrations of ammonium acetate $(5,10,20,50$, and $100 \mathrm{mM})$ at $120 \mathrm{~h}$ promoted CHA biosynthesis at different levels. The level of CHA production was improved when the concentration of ammonium acetate was increased from 5 to $50 \mathrm{mM}$. Specifically, the optimal concentration of ammonium acetate was $50 \mathrm{mM}$, where CHA production reached the maximum $(64.7 \mathrm{mg} / \mathrm{l})$ which was 2.2 fold higher than that in the non-treatment group. A $100 \mathrm{mM}$ concentration of ammonium acetate resulted in the CHA production decreasing to $41.9 \mathrm{mg} / \mathrm{l}$, indicating that a high content of ammonium acetate had a negative effect on CHA biosynthesis. Therefore, the optimized concentration of ammonium acetate supplementation for enhancing CHA production was $50 \mathrm{mM}$.

The feeding time of ammonium acetate also played an important role in $\mathrm{CHA}$ biosynthesis. The determinate ammonium acetate $(50 \mathrm{mM})$ was added at different times during the fermentation $(72,120,168,216,264$, and $312 \mathrm{~h}$ ) in the shake flasks (Fig. 2B). The production of $\mathrm{CHA}$ was first increased then decreased with feeding time, which reached a maximum $(62.0 \mathrm{mg} / \mathrm{l})$ at $120 \mathrm{~h}$. The promoting effect of ammonium acetate supplementation on CHA production at earlier times might be related to the beneficial effects of nutrient addition on fungal growth and product synthesis [28]. Overall, the optimal conditions for ammonium acetate supplementation for CHA biosynthesis was feeding $50 \mathrm{mM}$ ammonium acetate at $120 \mathrm{~h}$ after inoculation. Hence, the supplementation of ammonium acetate was an effective strategy for improving CHA production (Fig. 3).

\section{Effects of the Feeding Strategy on Metabolic Parameters}

The strategy of feeding ammonium acetate $(50 \mathrm{mM}$ at $120 \mathrm{~h}$ ) was confirmed to have a significant positive role in
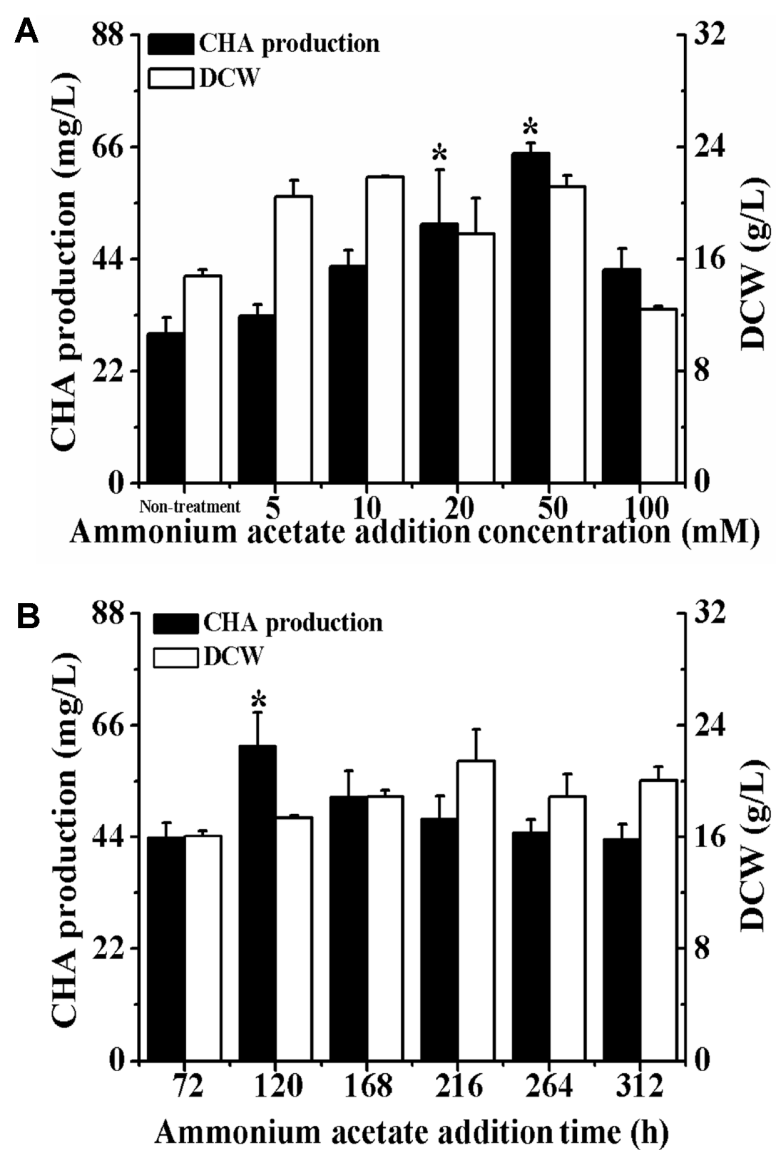

Fig. 2. Effects of additional concentration (A) and additional time (B) of ammonium acetate on chaetominine (CHA) production and dry cell weight (DCW) at $336 \mathrm{~h}(180 \mathrm{rpm}$, $28^{\circ} \mathrm{C}$ ) by Aspergillus fumigatus.

Non-treatment group: without addition of ammonium acetate. Experimental of additional concentration: 5, 10, 20, 50, and $100 \mathrm{mmol} / \mathrm{l}$. Experiment with additional time: 72, 120, 168, 216, 264, and $312 \mathrm{~h}$. ${ }^{*}$ Indicates statistical significance $(p<0.05)$ compared to the control group.

CHA biosynthesis. Thus, metabolic parameters (DCW, residual sugar, CHA production, and DAHP synthase activity) were further investigated during the fermentation process in a shake flask.

As shown in Fig. 4A, the trends of DCW in the feeding of ammonium acetate and the non-treatment groups were similar before $120 \mathrm{~h}$ culture time. Thereafter, the growth of cells in the group that was fed with ammonium acetate was higher than the non-treatment group. The maximal DCW in the test and non-treatment groups were 19.4 and $16.7 \mathrm{~g} / 1$ at $288 \mathrm{~h}$, respectively.

The consumption tendency of residual sugar continued to decline in both groups, while residual sugar was consumed 

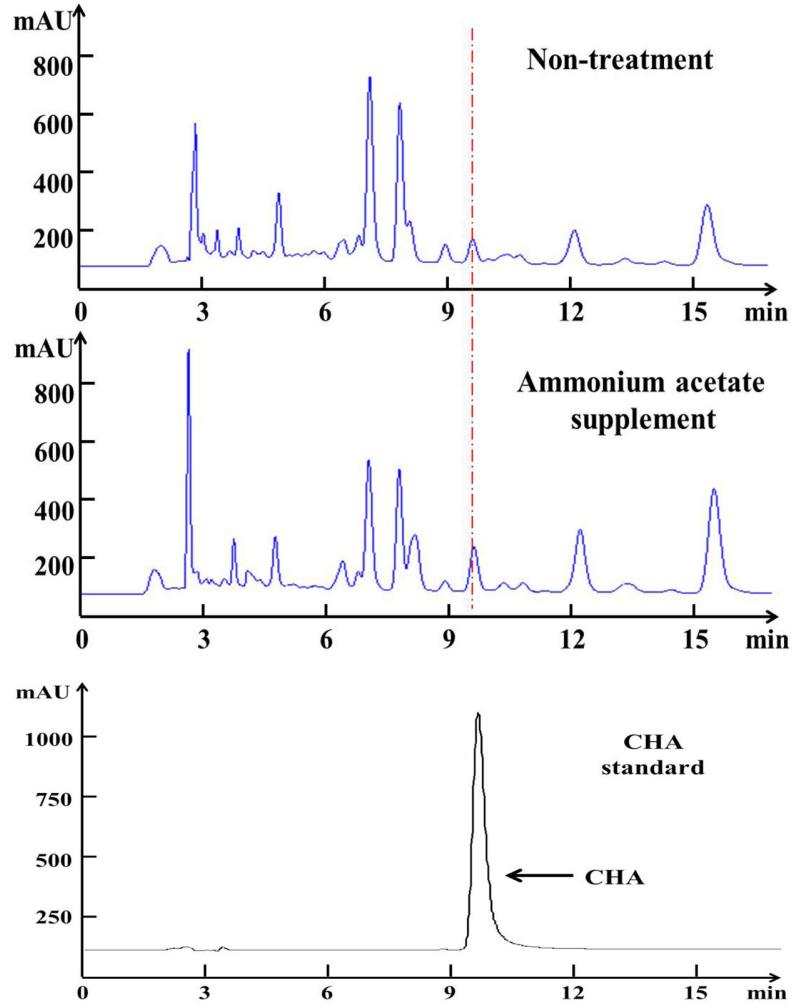

Fig. 3. HPLC chromatograms of CHA at $227 \mathrm{~nm}$ in the nontreatment group and ammonium acetate addition group $(50 \mathrm{mmol} / \mathrm{l}, 120 \mathrm{~h})$ at $336 \mathrm{~h}\left(180 \mathrm{rpm}, 28^{\circ} \mathrm{C}\right)$ by Aspergillus fumigatus.

Condition of HPLC: acetonitrile: water $=65: 35$, flow $=1 \mathrm{ml} / \mathrm{min}$, temperature $=25^{\circ} \mathrm{C}$, injection volume $=20 \mu \mathrm{l}$.

quickly after feeding of ammonium acetate (Fig. 4B). As shown in Fig. 4C, the level of CHA production was first increased and then decreased. Moreover, $\mathrm{CHA}$ production with the addition of ammonium acetate was 1.9 fold higher than that in non-treatment $(33.7 \mathrm{mg} / \mathrm{l})$. Moreover, the yield of CHA with ammonium acetate addition was higher than the non-treatment group in supplemental data 1, which meant the increasing CHA production appeared to be in the growth-dependent manner.

In previous research, we speculated and verified that the biosynthesis of CHA was through the shikimate pathway, and that DAHP synthase was a key enzyme in the process. Thus, the activity of DAHP synthase was investigated with ammonium acetate supplementation at different measured points of the fermentation process (Fig. 4D). The activity of DAHP synthase increased and then decreased in both the test and non-treatment groups. Importantly, the activity of DAHP synthase increased to a maximum, which was $52.6 \%$ above the non-treatment group at $240 \mathrm{~h}$. These data indicated that the strategy of ammonium acetate supplementation was beneficial for improving the biosynthesis of CHA.

Yang et al. also found that various fermentation parameters, such as the activities of key enzymes and the content of metabolites, were enhanced when either urea or ammonium chloride was used as the sole source of nitrogen [29]. It can clearly be seen that the strategy of feeding ammonium acetate could significantly promote the activity of key enzyme to optimize the fermentation parameters for enhancing CHA production.

\section{Gene Expression in Response to Ammonium Acetate Addition}

The feeding strategy we used was beneficial for cell growth and DAHP synthase activity to enhance CHA production. We next researched the mRNA transcript levels of laeA, dahp, cs and trpC using qRT-PCR to explore the mechanism of this effective strategy. The transcriptional levels of these key genes with supplementation of ammonium acetate were sampled at 48, 144, 240 and $336 \mathrm{~h}$ during the fermentation.

As shown in Fig. 5A, feeding ammonium acetate significantly increased the expression of laeA 4.2 fold and 2.4 fold at 144 and $336 \mathrm{~h}$, respectively, compared to the non-treatment group. The mRNA transcript levels of dahp were also improved remarkably (43.4 fold and 5.5 fold at 144 and $240 \mathrm{~h}$, respectively) with supplementation of ammonium acetate (Fig. 5B). The trend of trpC expression was similar with earlier results (Fig. 5D). In contrast, the influence of ammonium acetate addition was not significant on mRNA transcript level of cs (1.5- and 1.3- fold increases at 144 and $336 \mathrm{~h}$, respectively) (Fig. 5C). These results demonstrated that the supplementation of ammonium acetate could promote the mRNA transcript expression of key genes in the CHA synthesis pathway.

In general, an effective strategy for enhancing production of a target compound could affect mRNA or protein levels of regulated factors in the biosynthesis pathway of the target compound. For example, $\mathrm{Lu}$ et al. found the biosynthesis of dalesconols $\mathrm{A}$ and $\mathrm{B}$ was enhanced via calcium/calmodulin signaling with addition of $\mathrm{Ca}^{2+}$ [30]. In addition, Long et al. demonstrated that production of cellulase and hemicellulase was improved via the increased transcription factor, creA, in Trichoderma orientalis EU7-22 [31]. Moreover, Pandey and co-workers found that vindoline production was promoted by up-regulation of the Catharanthus AP2-domain protein and down-regulation of Cys2/His2-type zinc finger protein family transcriptional repressors [32]. 

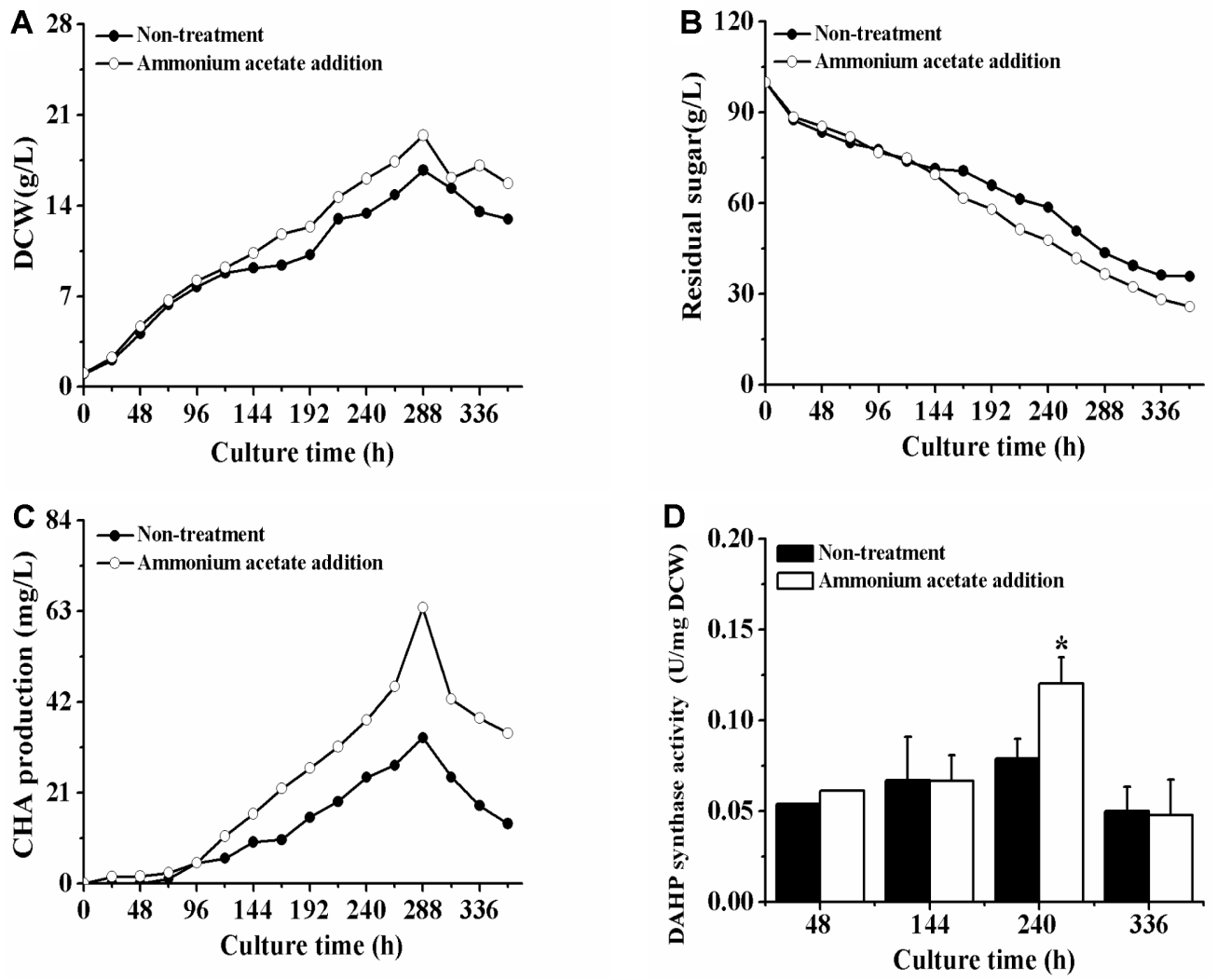

Fig. 4. Time courses of DCW (A), residual sugar (B), chaetominine production (C) and DAHP synthase activity (D) in shake flask with non-treatment and ammonium acetate feeding strategy.

Aspergillus fumigatus CY018 was cultured $360 \mathrm{~h}$ at $180 \mathrm{rpm}$ and $28^{\circ} \mathrm{C}$. ${ }^{*}$ Indicates statistical significance $(p<0.05)$ compared to the non-treatment group.

We researched the mRNA transcript levels of important genes/enzymes to reveal the effect of our strategy on CHA production. In the current work, feeding ammonium acetate increased the transcription levels of laeA, dahp and $\operatorname{trpC}$, which were key genes involved in DAHP synthase activity and CHA production. The activity of DAHP synthase was also improved by the feeding strategy. This meant supplementation of ammonium acetate positively regulated gene expression to improve enzyme activity. Therefore, the obtained results showed and verified that the strategy of ammonium acetate supplementation upregulated the transcription levels of key genes in the biosynthetic pathway to promote the biosynthesis of CHA by A. fumigatus CY018.

\section{Ammonium Acetate Feeding Strategy in a 5-L Bioreactor}

The feeding rate of nutrients is a helpful strategy in scaleup fermentation. Zhang et al. found that feeding glucose was beneficial for Antrodin C accumulation with $40 \mathrm{~g} / 1$ glucose in a 15-L bioreactor [33]. Therefore, for scaling up the fermentation by feeding ammonium acetate, the feeding rate $(0.01$ and $0.03 \mathrm{~g} / \mathrm{l} \cdot \mathrm{h})$ was investigated in a 5 -L stirred bioreactor compared to non-treatment (Fig. 6C). The time profiles of dissolved oxygen (DO), residual sugar, CHA production, $\mathrm{DCW}$, and $\mathrm{pH}$ were first investigated with a feeding rate of $0.03 \mathrm{~g} / \mathrm{l} \cdot \mathrm{h}$ (Fig. 6A).

The trend of DO was similar to non-treatment but declined slowly and maintained 0 from 216-312 h. Cell growth and CHA biosynthesis resulted in DO declining and cell death resulted in the DO rebound. DCW was also similar to non-treatment and the value of DCW with addition was higher than control. Residual sugar in the 5-L bioreactor was consumed rapidly compared to nontreatment and finally reached $11.3 \mathrm{~g} / 1$. CHA production in the 5-L bioreactor was increased to the maximum value of $46.99 \mathrm{mg} / \mathrm{l}$ at $312 \mathrm{~h}$ and then decreased. CHA concentration decreased towards the end of the fermentation which indicated metabolic instability for the target secondary 

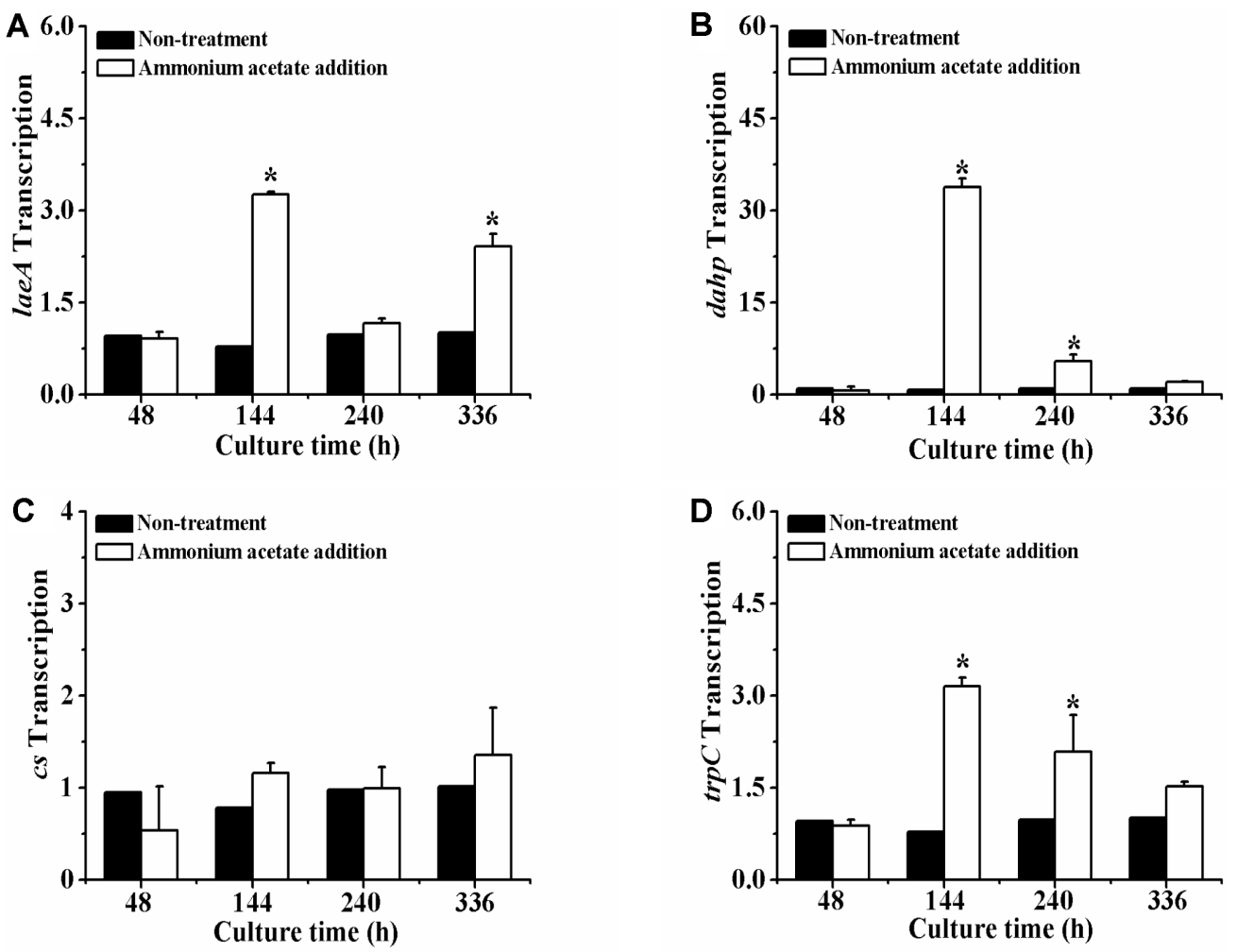

Fig. 5. Response of transcript level of genes related to chaetominine biosynthesis when feeding strategy and non-treatment were shown.

laeA as a global regulator (A), dahp as 3-Deoxy-arabino-heptulosonate-7-phosphate synthase (B), cs as chorismate synthase (C), and $\operatorname{trpC}$ as anthranilate synthase (D). Aspergillus fumigatus CY018 was cultured $360 \mathrm{~h}$ at $180 \mathrm{rpm}$ and $28^{\circ} \mathrm{C}$. *Indicates statistical significance $(p<0.05)$ compared to the non-treatment group.

metabolite. One known example of this was clavulanic acid $[34,35]$. The phenomenon demonstrated that novel routes in fermentations would open, resulting in a CHA decrease. The $\mathrm{pH}$ increased early during the fermentation process, then decreased and remained at approximately 4.0 earlier than non-treatment. This $\mathrm{pH}$ was beneficial to cell growth and target compound synthesis.

$\mathrm{CHA}$ production in the 5 - $\mathrm{L}$ bioreactor with a $0.03 \mathrm{~g} / \mathrm{l} \cdot \mathrm{h}$ feeding rate was lower than the results in the shake flasks $(62.10 \mathrm{mg} / \mathrm{l})$. This indicated that the rate of feeding ammonium acetate needed to be adjusted. A $0.01 \mathrm{~g} / \mathrm{l} \cdot \mathrm{h}$ rate of feeding ammonium acetate was applied in the $5-\mathrm{L}$ bioreactor (Fig. 6B). DO, residual sugar, and $\mathrm{pH}$ were all increased for a short time (96-144 h) after feeding, which was similar to non-treatment. The trend of DCW was greater at the $0.03 \mathrm{~g} / \mathrm{l} \cdot \mathrm{h}$ rate. Moreover, $\mathrm{CHA}$ production reached $57.9 \mathrm{mg} / \mathrm{l}$, which was closer to the $62.10 \mathrm{mg} / 1$ in the shake flask than the $0.03 \mathrm{~g} / \mathrm{l} \cdot \mathrm{h}$ rate and non-treatment $(46.11 \mathrm{mg} / \mathrm{l})$. This information meant that a $0.01 \mathrm{~g} / \mathrm{l} \cdot \mathrm{h}$ rate of feeding ammonium acetate was more beneficial for CHA biosynthesis than a $0.03 \mathrm{~g} / \mathrm{l} \cdot \mathrm{h}$ rate and non-treatment in the 5-L bioreactor. Moreover, the detailed comparison of each parameter (DO, residual sugar, chaetominine production, $\mathrm{DCW}$, and $\mathrm{pH}$ ) was shown in the supplemental data 2.

Hence, the fermentation of $A$. fumigatus CY018 for producing CHA was successfully scaled up with a feeding $0.01 \mathrm{~g} / \mathrm{l} \cdot \mathrm{h}$ rate of ammonium acetate in the 5 - $\mathrm{L}$ bioreactor. The results of the current work indicated that the supplementation strategy of ammonium acetate was beneficial for CHA biosynthesis by A. fumigatus CY018. Meanwhile, this work served as a valuable experience for enhancing target compound production in submerged fermentation.

Finally, in this paper, the supplementation of selected ammonium acetate proved beneficial for CHA biosynthesis. The optimization of additional concentrations and fermentation times of ammonium acetate was first performed in the shake flask. Maximal CHA production $(62.1 \mathrm{mg} / \mathrm{l})$ was achieved with addition of $50 \mathrm{mM}$ ammonium acetate at $120 \mathrm{~h}$ of fermentation. The activity of key enzyme and 

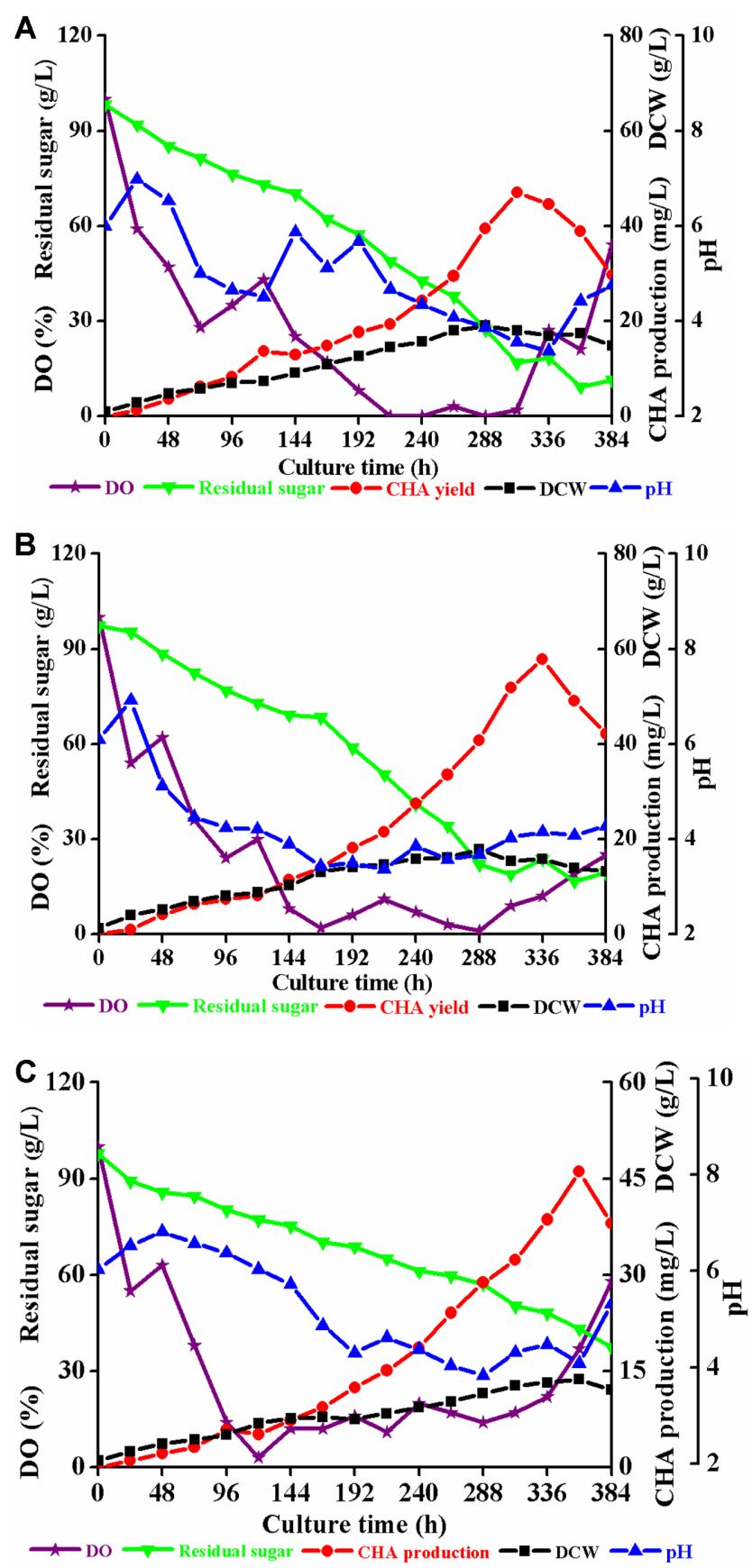

Fig. 6. Time courses of DO, chaetominine production, residual sugar, $\mathrm{pH}$, and DCW in Aspergillus fumigatus cultured in a 5-L bioreactor with feeding rates of $0.03 \mathrm{~g} / \mathrm{l} \cdot \mathrm{h}(\mathbf{A}), 0.01 \mathrm{~g} / \mathrm{l} \cdot \mathrm{h}(\mathbf{B})$ and non-treatment $(\mathbf{C})$.

The culture condition of A. fumigatus CY018 was $300 \mathrm{rpm}, 28^{\circ} \mathrm{C}$ and $1.5 \pm 0.1$ vessel volume $/ \mathrm{min}$ of air flow. The $11.55 \mathrm{~g}$ of ammonium acetate $(50 \mathrm{mM}, 3 \mathrm{~L}$ broth) was supplied at $120 \mathrm{~h}$.

transcription levels of key genes (laeA, dahp, and $\operatorname{trpC}$ ) were promoted with a supplementation strategy using ammonium acetate, which enhanced CHA production ultimately. Moreover, the feeding rate of ammonium acetate was researched and determined to be $0.01 \mathrm{~g} / 1 \cdot \mathrm{h}$ in a $5-\mathrm{L}$ bioreactor. These results demonstrated that supplementation of ammonium acetate was a significant strategy to improve the production of CHA. Furthermore, this work served as a valuable experience for promoting a target compound from marine fungus by supplementation of effective nutrients in submerged fermentation.

\section{Acknowledgments}

This work was sponsored by the National Natural Science Foundation of China (81741156), the Shanghai Sailing Program (17YF1403700), and the Fundamental Research Funds for the Central Universities (222201714023, 22221818014).

\section{Conflict of Interest}

The authors have no financial conflicts of interest to declare.

\section{References}

1. Sashidhara KV, White KN, Crews P. 2009. A Selective Account of effective paradigms and significant outcomes in the discovery of inspirational marine natural products. $J$. Nat. Prod. 72: 588-603.

2. Liao L, Bae SY, Won TH, You M, Kim SH, Oh DC, et al. 2017. Asperphenins A and B, lipopeptidyl benzophenones from a marine-derived Aspergillus sp. Fungus. Org. Lett. 19: 2066-2069.

3. Wang X, Yu H, Zhang Y, Lu X, Wang B, Liu X. 2018. Bioactive pimarane-type diterpenes from marine organisms. Chem. Biodivers. 15: e1700276.

4. Duarte K, Rocha-Santos TAP, Freitas AC, Duarte AC. 2012. Analytical techniques for discovery of bioactive compounds from marine fungi. Trends Analyt. Chem. 34: 97-110.

5. Lindequist U. 2016. Marine-derived pharmaceuticals challenges and opportunities. Biomol. Ther. 24: 561-571.

6. Zhang XY, Bao J, Zhong J, Xu XY, Nong XH, Qi SH. 2013. Enhanced production of a novel cytotoxic chromone oxalicumone A by marine-derived mutant Penicillium oxalicum SCSIO 24-2. Appl. Microbiol. Biotechnol. 97: 9657-9663.

7. Zhang G, Li J, Zhu T, Gu Q, Li D. 2016. Advanced tools in marine natural drug discovery. Curr. Opin. Biotechnol. 42: 13-23.

8. Agrawala S, Adholeya A, Barrow CJ, Deshmukh SK. 2018. Marine fungi: an untapped bioresource for future cosmeceuticals. Phytochem. Lett. 23: 15-20.

9. Li XJ, Zhang Q, Zhang AL, Gao JM. 2012. Metabolites from Aspergillus fumigatus, an endophytic fungus associated with 
Melia azedarach, and their antifungal, antifeedant, and toxic activities. J. Agric. Food Chem. 60: 3424-3431.

10. Li YX, Himaya SW, Dewapriya P, Zhang C, Kim SK. 2013. Fumigaclavine $\mathrm{C}$ from a marine-derived fungus Aspergillus Fumigatus induces apoptosis in MCF-7 breast cancer cells. Mar. Drugs 11: 5063-5086.

11. Yao JY, Jiao RH, Liu CQ, Zhang YP, Yu WG, Lu YH, et al. 2016. Assessment of the cytotoxic and apoptotic effects of Chaetominine in a human leukemia cell line. Biomol. Ther. 24: 147-155.

12. Yao JY, Wei X, Lu YH. 2016. Chaetominine reduces MRP1mediated drug resistance via inhibiting PI3K/Akt/Nrf2 signaling pathway in K562/Adr human leukemia cells. Biochem. Biophys. Res. Commun. 473: 867-873.

13. Stanley A, Punil Kumar HNP, Mutturi S, Vijayendra SVN. 2018. Fed-batch strategies for production of PHA using a native isolate of Halomonas venusta KT832796 strain. Appl. Biochem. Biotechnol. 184: 935-952.

14. Wang DH, Chen FF, Wei GY, Jiang M, Dong MS. 2015. The mechanism of improved pullulan production by nitrogen limitation in batch culture of Aureobasidium pullulans. Carbohydr. Polym. 127: 325-331.

15. Wang XYZ, Dong JJ, Xu GC, Han RZ, Ni Y. 2016. Enhanced curdlan production with nitrogen feeding during polysaccharide synthesis by Rhizobium radiobacter. Carbohydr. Polym. 150: 385-391.

16. Liu CQ, Pan ZH, An FL, Lu YH. 2018. Co-addition strategy for enhancement of chaetominine from submerged fermentation of Aspergillus fumigatus CY018. Appl. Biochem. Biotechnol. 186: 384-399.

17. Chludzinski AM, Salter DS, Nasser D. 1972. Feedback regulation of 3-deoxy-D-arabino-heptulosonate 7-phosphate synthetase from a marine bacterium, Vibrio MB22. J. Bacteriol. 109: 1162-1169.

18. Hu PS, Meng Y, Wise RP. 2009. Functional contribution of chorismate synthase, anthranilate synthase, and chorismate mutase to penetration resistance in Barley-Powdery mildew interactions. Mol. Plant Microbe. Interact. 22: 311-320.

19. Culbertson JE, Chung DH, Ziebart KT, Espiritu E, Toney MD. 2015. Conversion of aminodeoxychorismate synthase into anthranilate synthase with janus mutations: mechanism of pyruvate elimination catalyzed by chorismate enzymes. Biochemistry 54: 2372-2384.

20. Bok JW, Keller NP. 2004. LaeA, a regulator of secondary metabolism in Aspergillus spp. Eukaryot Cell 3: 527-535.

21. Xie WP, Ye LD, Lv XM, Xu HM, Yu HW. 2015. Sequential control of biosynthetic path ways for balanced utilization of metabolic intermediates in Saccharomyces cerevisiae. Metab. Eng. 28: 8-18.

22. Kong M, Wang FJ, Tian LY, Tang H, Zhang LP. 2018. Functional identification of glutamate cysteine ligase and glutathione synthetase in the marine yeast Rhodosporidium diobovatum. Naturwissenschaften 105: 4-12.
23. Zhao W, Xu JW, Zhong JJ. 2011. Enhanced production of ganoderic acids in static liquid culture of Ganoderma lucidum under nitrogen-limiting conditions. Bioresour. Technol. 102: 8185-8190.

24. Zhang YP, Jiao RH, Lu YH, Yao LY. 2016. Improvement of chaetominine production by tryptophan feeding and medium optimization in submerged fermentation of Aspergillus fumigatus CY018. Bioresour. Bioprocess. 3: 45-53.

25. Zhu YX, Yao LY, Jiao RH, Lu YH, Tan RX. 2014. Enhanced production of Fumigaclavine $\mathrm{C}$ in liquid culture of Aspergillus fumigatus under a two-stage process. Bioresour. Technol. 152: 162-168.

26. Liu CQ, Jiao RH, Yao LY, Zhang YP, Lu YH, Tan RX. 2016. Adsorption characteristics and preparative separation of chaetominine from Aspergillus fumigatus mycelia by macroporous resin. J. Chromatogr. B. 1015: 135-141.

27. Li SB, Liu LM, Chen J. 2015. Mitochondrial fusion and fission are involved in stress tolerance of Candida glabrata. Bioresour. Bioprocess 2: 12-20.

28. Lindlbauer KA, Marx H, Sauer M. 2017. Effect of carbon pulsing on the redox household of Lactobacillus diolivorans in order to enhance 1,3-propanediol production. N. Biotechnol. 34: 32-39.

29. Yang SX, Chen XL, Xu N, Liu LM, Chen J. 2014. Urea enhances cell growth and pyruvate production in Torulopsis glabrata. Biotechnol. Prog. 30: 19-27.

30. Lu YH, Pan ZH, Tao J, An FA. 2018. Induced effect of $\mathrm{Ca}^{2+}$ on dalesconols $\mathrm{A}$ and $\mathrm{B}$ biosynthesis in the culture of Daldinia eschscholzii via calcium/calmodulin signaling. J. Biosci. Bioeng. 125: 205-210.

31. Long CN, Cheng YJ, Cui JJ, Liu J, Gan LH, Zeng B, Long MN. 2018. Enhancing cellulase and hemicellulase production in Trichoderma orientalis EU7-22 via knockout of the creA. Mol. Biotechnol. 60: 55-61.

32. Pandey SS, Singh S, Babu CSV, Shanker K, Srivastava NK, Shukla AK, et al. 2016. Fungal endophytes of Catharanthus roseus enhance vindoline content by modulating structural and regulatory genes related to terpenoid indole alkaloid biosynthesis. Sci. Rep. 6: 26583-26596.

33. Zhang H, Hu YD, Lu RQ, Xia YJ, Zhang BB, Xu GR. 2014. Integrated strategy of $\mathrm{pH}$-shift and glucose feeding for enhanced production of bioactive Antrodin C in submerged fermentation of Antrodia camphorate. J. Ind. Microbiol. Biotechnol. 41: 1305-1310.

34. Roubos JA, Krabben P, de Laat WT, Babuska R, Heijnen JJ. 2002. Clavulanic acid degradation in Streptomyces clavuligerus fed-batch cultivations. Biotechnol. Prog. 18: 451-457.

35. Mayer AF, Deckwer WD. 1996. Simultaneous production and decomposition of clavulanic acid during Streptomyces clavuligerus cultivations. Appl. Microbiol. Biotechnol. 45: 41-46. 